\title{
Stable clinical course in three siblings with late-onset isolated sulfite oxidase deficiency: a case series and literature review
}

Maoqiang Tian ${ }^{1,2,3}, Y_{i} \mathrm{Qu}^{1,2^{*}}$ (D), Lingyi Huang ${ }^{4}$, Xiaojuan $\mathrm{Su}^{1,2}$, Shiping $\mathrm{Li}^{1,2}$, Junjie Ying ${ }^{1,2}$, Fengyan Zhao ${ }^{1,2}$ and Dezhi $\mathrm{Mu}^{1,2}$

\begin{abstract}
Background: Isolated sulfite oxidase deficiency (ISOD) is an autosomal recessive disorder caused by a deficiency of sulfite oxidase, which is encoded by the sulfite oxidase gene (SUOX). Clinically, the disorder is classified as one of two forms: the late-onset mild form or the classic early-onset form. The latter is life-threatening and always leads to death during early childhood. Mild ISOD cases are rare and may benefit from dietary therapy. To date, no cases of ISOD have been reported to recover spontaneously. Here, we present three mild ISOD cases in one family, each with a stable clinical course and spontaneous recovery.

Case presentation: All three siblings had two novel compound heterozygous mutations in the SUOX gene (NM_000456; c.1096C > T [p.R366C] and c.1376G > A [p.R459Q]). The siblings included two males and one female with late ages of onset (12-16 months) and presented with specific neuroimaging abnormalities limited to the bilateral globus pallidus and substantia nigra. The three patients had decreased plasma homocysteine levels. They exhibited a monophasic clinical course continuing up to 8.5 years even without dietary therapy.
\end{abstract}

Conclusion: This is the first report of mild ISOD cases with a stable clinical course and spontaneous recovery without dietary therapy. Our study provides an expansion for the clinical spectrum of ISOD. Furthermore, we highlight the importance of including ISOD in the differential diagnosis for patients presenting with late-onset symptoms, bilaterally symmetric regions of abnormal intensities in the basal ganglia, and decreased plasma homocysteine levels.

\section{Background}

Isolated sulfite oxidase deficiency (ISOD, OMIM 272300) is an inborn error of metabolism characterized by severe neurological impairment including dystonia severe psychomotor delay, refractory seizures, and lens dislocation. Clinically, the disorder is classified as one of two forms: mild or severe. Severe ISOD often presents within the first $72 \mathrm{~h}$ of life and leads to death during early childhood in

\footnotetext{
*Correspondence: quyi712002@163.com

'Department of Pediatrics, West China Second University Hospital, Sichuan University, Chengdu 610041, China

${ }^{2}$ Key Laboratory of Birth Defects and Related Diseases of Women and Children, (Sichuan University), Ministry of Education, Chengdu 610041, China Full list of author information is available at the end of the article
}

most cases [1,2]. ISOD is caused by a mutation in the sulfite oxidase gene $(S U O X)$ [2]. Sulfite oxidase (SUOX) is located in the intermembrane space of the mitochondria and catalyzes the degradation of sulfur-containing amino acids [3-5]. Mutations in the SUOX gene result in a decrease in SUOX activity, leading to an accumulation of sulfur-containing amino acids, inducing mitochondrial impairment and energy failure, and resulting in severe neurological damage [2]. Effective treatment is not available for most patients with ISOD [6], but clinical and biochemical improvements were observed in six mild ISOD cases treated with dietary therapy involving restricting the intake of methionine, cysteine, and taurine [7-11]. Therefore, early identification of ISOD, especially the mild type,

(c) The Author(s). 2019 Open Access This article is distributed under the terms of the Creative Commons Attribution 4.0 International License (http://creativecommons.org/licenses/by/4.0/), which permits unrestricted use, distribution, and reproduction in any medium, provided you give appropriate credit to the original author(s) and the source, provide a link to the Creative Commons license, and indicate if changes were made. The Creative Commons Public Domain Dedication waiver (http://creativecommons.org/publicdomain/zero/1.0/) applies to the data made available in this article, unless otherwise stated. 
may allow patients to benefit from dietary therapy. To date, no cases of ISOD have been reported to recover spontaneously. We report three cases of mild ISOD which experienced spontaneous recovery and maintained a stable clinical course even 8.5 years into follow-up.

\section{Case presentation}

The three patients in this report were children of a nonconsanguineous Chinese couple (Fig. 1a) who were born at term and followed a normal delivery. Their psychomotor developmental milestones were normal prior to disease onset. The main biochemical findings of each patient are summarized in Table 1. This study was approved by the Ethics committee of the Affiliated Hospital of Zunyi Medical University, China.

\section{Case 1}

Before age 1, psychomotor development in the firstborn male patient was normal, and he could walk independently. This patient exhibited rapid regression of acquired motor skills and cognition after a 2-day episode of mild diarrhea at age 1 . Seizures were not observed. On examination, his growth was found to be in the 90th percentile, and his alertness and eye movement were both normal. He was not able to sit. No dysmorphic features, tremor, ataxia, or involuntary movements were observed. Cranial nerve examination findings were normal. He had generalized hypotonia with normal deep tendon reflexes. His blood lactate level was slightly increased, but uric acid, plasma glucose, and cerebrospinal fluid (CSF) test results were unremarkable. Brain magnetic resonance imaging (MRI) using T2-weighted (T2WI) and fluid-attenuated inversion recovery (FLAIR) sequencing demonstrated high signal in lesions limited to the bilateral globus pallidus and substantia nigra (Fig. 2a). The patient received supportive and physical therapy. He could walk without support at age 3. Comparison of MR images obtained at 1 year after onset and 5.5 years after onset showed that the basal ganglia and substantia nigra

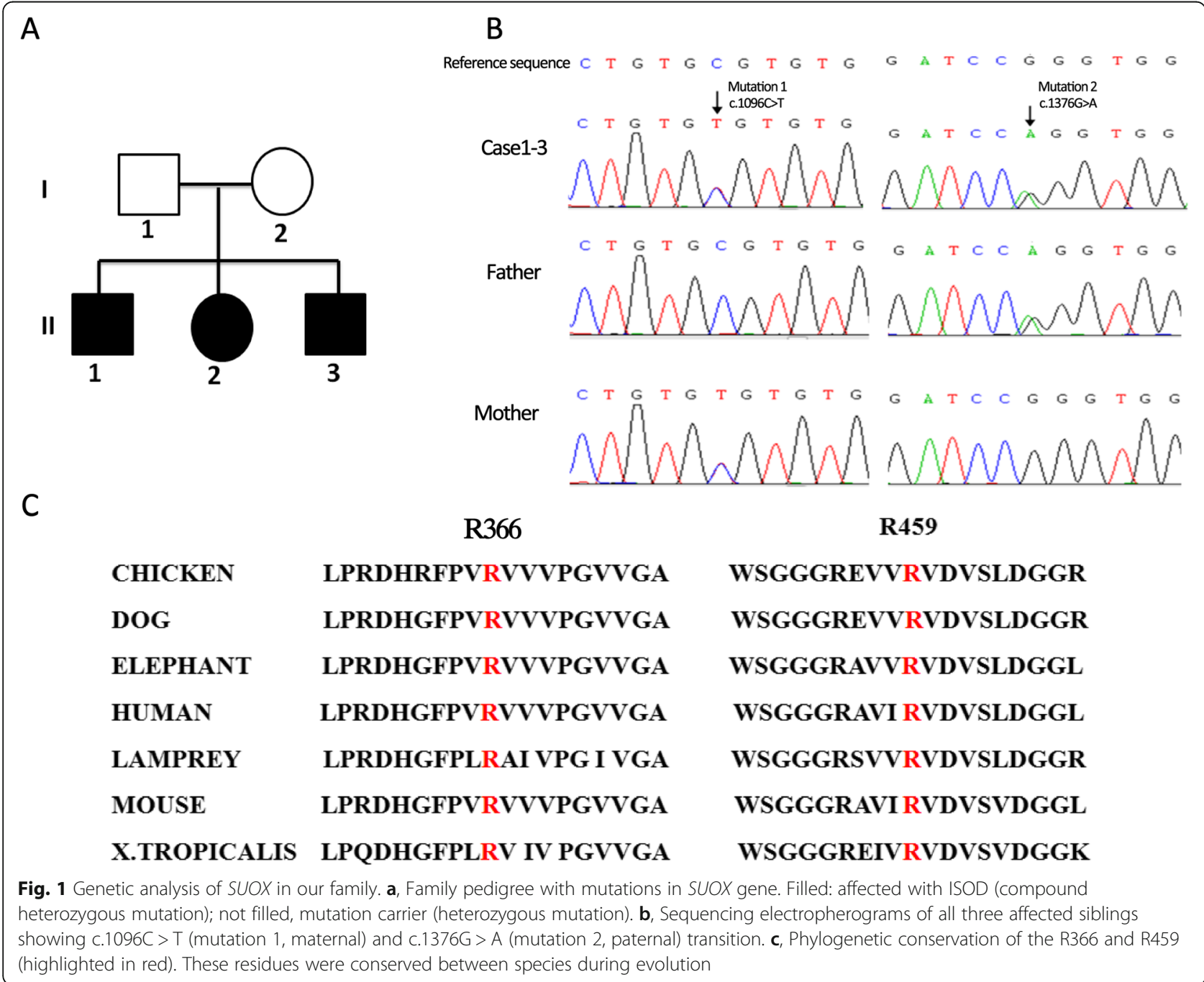


Table 1 Main biochemical findings of the three patients in this study with late-onset mild ISOD

\begin{tabular}{|c|c|c|c|c|}
\hline Parameter & Case 1 & Case 2 & Case 3 & Normal value \\
\hline Urine sulfite reaction (age) & Positive (9 years) & Positive (5 years) & Positive(4 years) & Negative \\
\hline Urine sulfite level (age) & $\begin{array}{l}45 \mathrm{mg} / \mathrm{L} \text { (9 years) } \\
50 \mathrm{mg} / \mathrm{L} \text { (9years and } 3 \text { months) }\end{array}$ & $\begin{array}{l}40 \mathrm{mg} / \mathrm{L} \text { ( } 5 \text { years) } \\
30 \mathrm{mg} / \mathrm{L} \text { ( } 5 \text { years and } 3 \text { months) }\end{array}$ & $\begin{array}{l}100 \mathrm{mg} / \mathrm{L} \text { ( } 4 \text { years) } \\
25 \mathrm{mg} / \mathrm{L}(4 \text { years and } 3 \text { months) }\end{array}$ & $<15 \mathrm{mg} / \mathrm{L}$ \\
\hline Homocysteine (age) & 3.74 mol/L (9 years) & $3.17 \mu \mathrm{mol} / \mathrm{L}$ (5 years) & $\begin{array}{l}2.48 \mu \mathrm{mol} / \mathrm{L} \text { (16 months) } \\
2.66 \mu \mathrm{mol} / \mathrm{L} \text { (4 years) }\end{array}$ & $5-15 \mu \mathrm{mol} / \mathrm{L}$ \\
\hline Uric acid (age) & 255 mol/L (1 year) & 269 umol/L (14 months) & 400 umol/L (16 months) & $208-428 \mu \mathrm{mol} / \mathrm{L}$ \\
\hline Blood lactate (age) & $2.4 \mathrm{mmol} / \mathrm{L}$ (1 year) & 5 mmol/L (14 months) & $2.48 \mathrm{mmol} / \mathrm{L}$ (16 months) & $<2 \mathrm{mmol} / \mathrm{L}$ \\
\hline \multicolumn{5}{|l|}{ Cerebrospinal fluid results } \\
\hline Cell count (age) & 10 cells/ $\mu$ l (1 year) & 5 cells/ $\mu$ ( 14 months) & 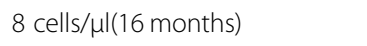 & $<10$ cells $/ \mu$ l \\
\hline Protein (age) & 342 mg/L (1 year) & 159 mg/L (14 months) & 269 mg/L(16 months) & $<450 \mathrm{mg} / \mathrm{L}$ \\
\hline Glucose (age) & $3.2 \mathrm{mmol} / \mathrm{L}$ (1 year) & 3.44 mmol/L (14 months) & 2.95 mmol/L(16 months) & $>2.8 \mathrm{mmol} / \mathrm{L}$ \\
\hline
\end{tabular}

lesions had almost disappeared (Fig. 2b and c). During 8.5 years of follow-up, no recurrent episodes occurred. His performance in school was normal. His growth was in the 90th percentile (height, $134 \mathrm{~cm}$; weight, $26 \mathrm{~kg}$; and head circumference, $54 \mathrm{~cm}$ ). He had an unsteady gait (Additional file 1: Video S1), mild spasticity of the lower limbs, and brisk symmetrical tendon reflexes. Ophthalmic examination results were normal. Mental development measured using the Wechsler Mental Development Scale-Revised showed a borderline level of mental development, with scores in the 90th percentile.
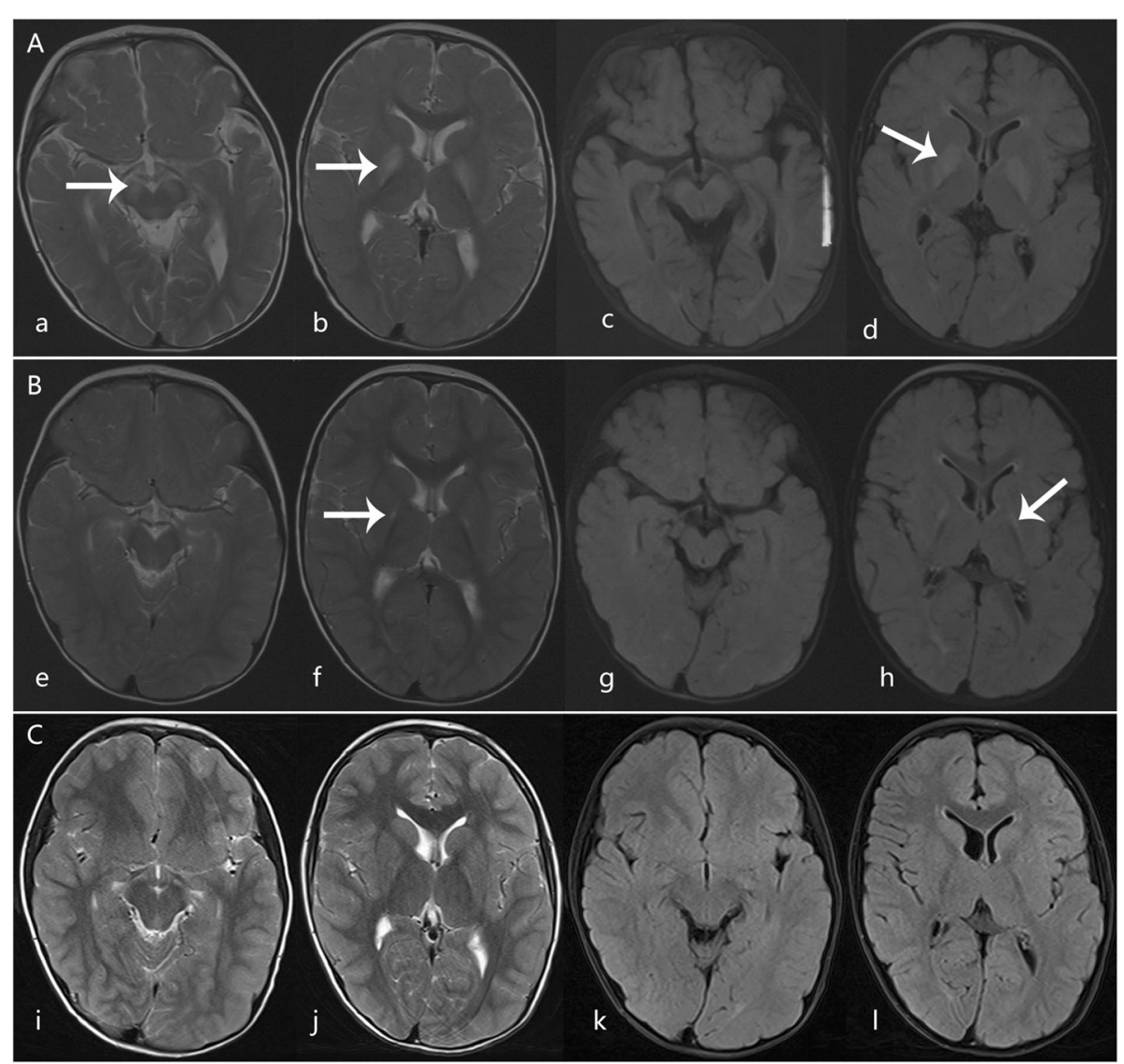

Fig. 2 Axial T2-weighted imaging (T2WI) and fluid-attenuated inversion recovery (FLAIR) magnetic resonance imaging (MRI) scans from Case 1. a, b, T2 W1 and $\mathbf{c}$, d, FLAIR performed 3 days after disease onset at age 1, showing high signal in the bilateral globus pallidus and substantia nigra (arrows). e-h, Follow-up images 1 year after onset revealed that hyper-signal lesions significantly shrank (arrows). $\mathbf{i}-\mathbf{l}$, Lesions on the basal ganglia had almost disappeared and the lesion on the substantia nigra disappeared 5.5 years after the onset 


\section{Case 2}

This patient was the couple's second child. She developed lethargy and somnolence and experienced one generalized brief seizure at 14 months after a 4-day episode of mild diarrhea. Her alertness was mildly decreased. Neurologic examination revealed severe generalized hypotonia and an inability to control her head movement. Cranial nerve examination was normal. She had normal deep tendon reflexes. Her blood lactate level was $5 \mathrm{mmol} / \mathrm{L}$, and plasma uric acid was $269 \mu \mathrm{mol} / \mathrm{L}$. The CSF test results indicated that cell counts, protein levels, and glucose levels were normal. T2-WI and FLAIR brain MRI scans revealed high signal lesions limited to the bilateral globus pallidus and the substantia nigra (Fig. 3a). She reacquired head control at age 18 months. Repeat MRI of the brain was performed at 1.5 years after onset. The results showed that the original lesions had shrunk significantly (Fig. 3b). At 5.5 years of age, she could walk several steps with an unsteady gait. She had some choreiform movements, which became more evident while walking (Additional file 2: Video S2). Her vocabulary was normal (Additional file 3: Video D3). Her growth was in the 90th percentile (height, $110 \mathrm{~cm}$; weight, $18 \mathrm{~kg}$; head circumference, $50 \mathrm{~cm}$ ). Gesell Developmental Observation-Revised screening was performed to observe her behavior. The results showed a severe delay in gross motor development and a mild delay in language and social-emotional responses. Ophthalmic examination results were normal.

\section{Case 3}

At age 16 months, the second male child was hospitalized due to acute onset of regression of motor and mental skills and choreoathetoid movements. We did not find any prodromal illness prior to the onset of disease. The neurologic examination revealed generalized hypotonia with tendon hyperreflexia and an inability to sit and roll over. Cranial nerve examination findings were normal. T2WI and FLAIR brain MRI scans revealed high signal in the bilateral globus pallidus and substantia nigra. Diffusion-weighted imaging through the basal ganglia revealed hyperintensity of the globus pallidus (Fig. 4a). The patient's blood lactate level was $2.48 \mathrm{mmol} / \mathrm{L}$, and his homocysteine level was $2.48 \mu \mathrm{mol} / \mathrm{L}$. Other routine biological parameters, including uric acid levels, were normal. CSF test results were unremarkable (Table1). Symptomatic and supportive therapies improved his symptoms. He was readmitted to the hospital due to a deterioration in movement control following a mild upper respiratory infection 11 days after discharge (20 days after disease onset). Repeat brain MRI revealed additional conspicuous lesions, and T2-weighted and diffusion-weighted imaging uncovered necrotic lesions on the left globus pallidus (Fig. $4 \mathrm{~b})$. The patient's hypotonia progressed to generalized hypertonia over the clinical course. Follow-up MRI scans at 2.5 years after disease onset revealed that the original lesions were smaller (Fig. 4c). Head control was reacquired at age 2 . The patient could not sit until 4.5 years of age because of generalized hypertonia. He had some mild choreiform movements (Additional file 4: Video S4). He could only speak a few words but had good language comprehension. His growth was in the 90th percentile (head circumference, $46 \mathrm{~cm}$; height, $100 \mathrm{~cm}$; and weight, $15 \mathrm{~kg}$ ). Gesell Developmental Observation-Revised screening was performed, and the results showed a severe delay in gross motor development, in fine motor quotient, and a

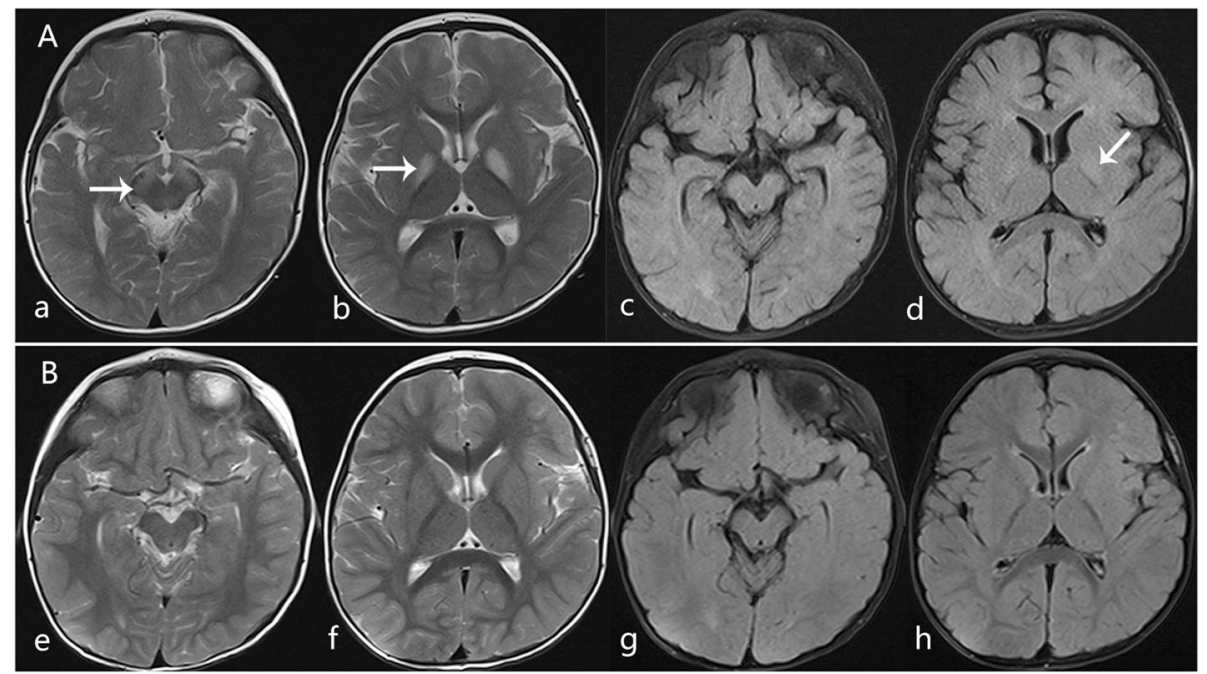

Fig. 3 Axial T2WI and FLAIR MRI scans from Case 2. a-d, MRI abnormalities observed 2 days after disease onset at 14 months. T2WI (a, b) and FLAIR (c, d) showed high signal in the bilateral globus pallidus and substantia nigra (arrows). e-h, Follow-up images 1.5 years after onset revealed that the original lesions on the basal ganglia and substantia nigra had almost disappeared 

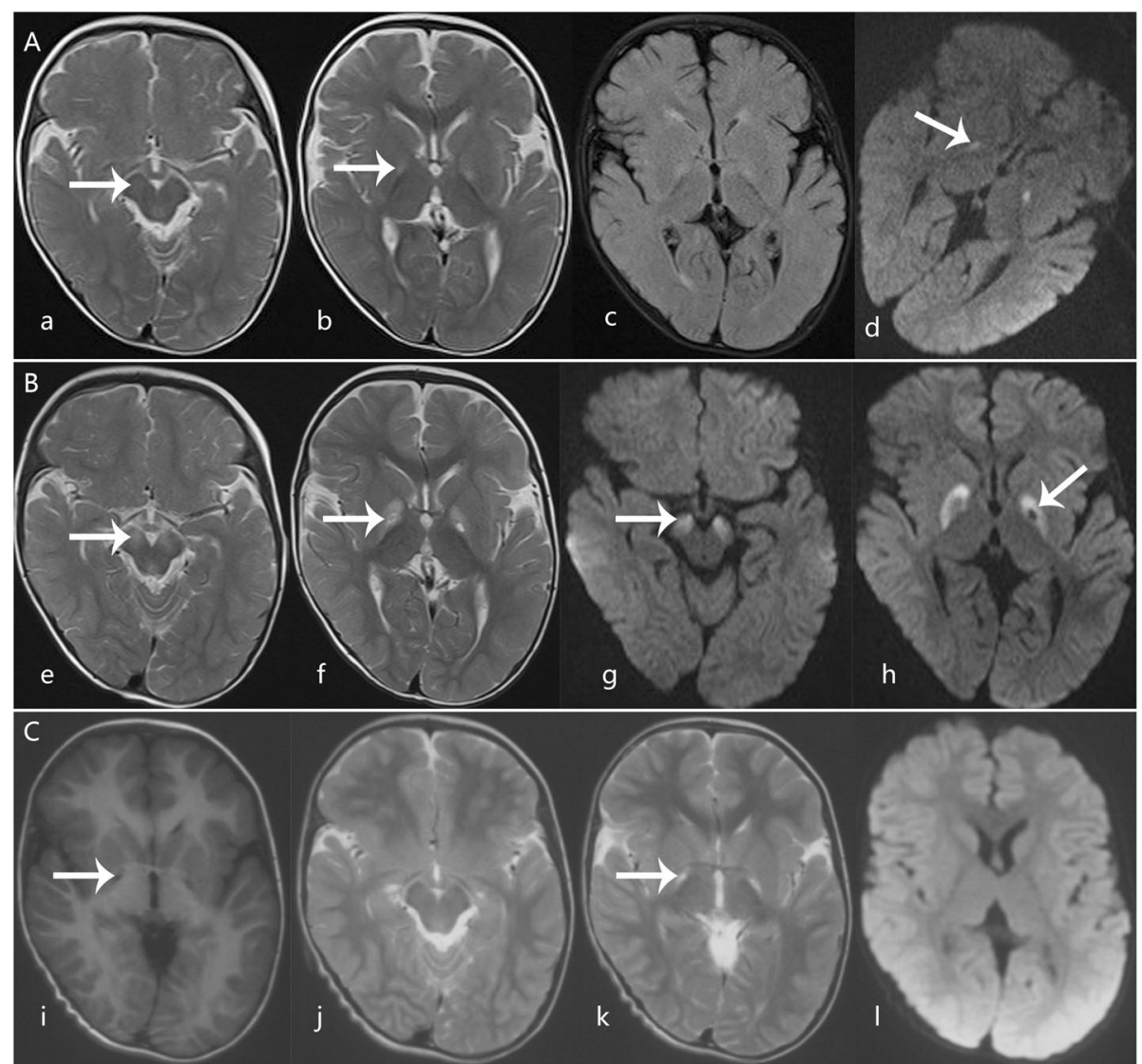

Fig. 4 Axial MRI scans from Case 3. T2WI (a, b), FLAIR (c), and diffusion-weighted imaging (DWI) (d) performed at 3 days after disease onset at age 16 months showing high signal in the bilateral globus pallidus and substantia nigra (arrows). e-h, Follow-up images 20 days after clinical onset revealed more conspicuous lesions than the original scan, with a necrotic lesion on the left globus pallidus on T2-weighted (e, $\mathbf{f}$ ) and diffusion-weighted imaging $(\mathbf{g}, \mathbf{h})$ (arrows). i-I, Follow-up images at 2.5 years after onset revealed that lesions on the substantia nigra disappeared (j) and lesions on the globus pallidus were smaller, with well-delineated cysts (i, k) (arrows) and without new lesions and brain atrophy

moderate delay in language and social-emotional responses. The patient's ophthalmic examination results were normal.

\section{Genetic findings}

Physicians suspected Leigh syndrome for Case 1. Genetic sequencing did not reveal the 3243A $>\mathrm{G}, 8344 \mathrm{~A}>\mathrm{G}$, $8993 \mathrm{~A}>\mathrm{G}$, and $8993 \mathrm{~T}>\mathrm{C}$ mutations. Furthermore, although Case 2 was suspected as succinic semialdehyde dehydrogenase deficiency, genetic sequencing showed no succinic semialdehyde dehydrogenase gene mutation. Therefore, whole-exome sequencing analysis was performed for this family when the eldest child was 9 years of age (the second child was 5 years old, and the third child was 4 years old). Whole-exome sequencing showed that all three siblings harbored two novel compound heterozygous mutations (NM_000456; c.1096C > T [p.R366C] and c.1376G > A [p.R459Q]) in the SUOX gene. We confirmed that $\mathrm{c} .1096 \mathrm{C}>\mathrm{T}$ came from the maternal line and that c.1376G > A came from the paternal line by Sanger sequencing (Fig. 1b). These mutations are likely pathogenic according to the guidelines for sequence variants provided by the American College of Medical Genetics and Genomics [12]. Residues R366and R459 of SUOX are conserved during evolution (Fig. 1c). Three prediction programs (PolyPhen2, SIFT and Mutation Taster) predicted that the mutations could affect the function of SUOX.

SUOX is associated with ISOD, and therefore, the levels of sulfite in the urine and plasma homocysteine were measured for each of the three children. This indicated that sulfite concentrations were higher than normal and that homocysteine levels were lower than normal in all three children (Table 1). The three siblings were eventually diagnosed with ISOD. Dietary therapy was not used due to the delayed diagnosis as well as due to economic limitations.

\section{Discussion and conclusions}

Very few patients present with the mild form of ISOD. Previously, only seven patients with mild ISOD have 


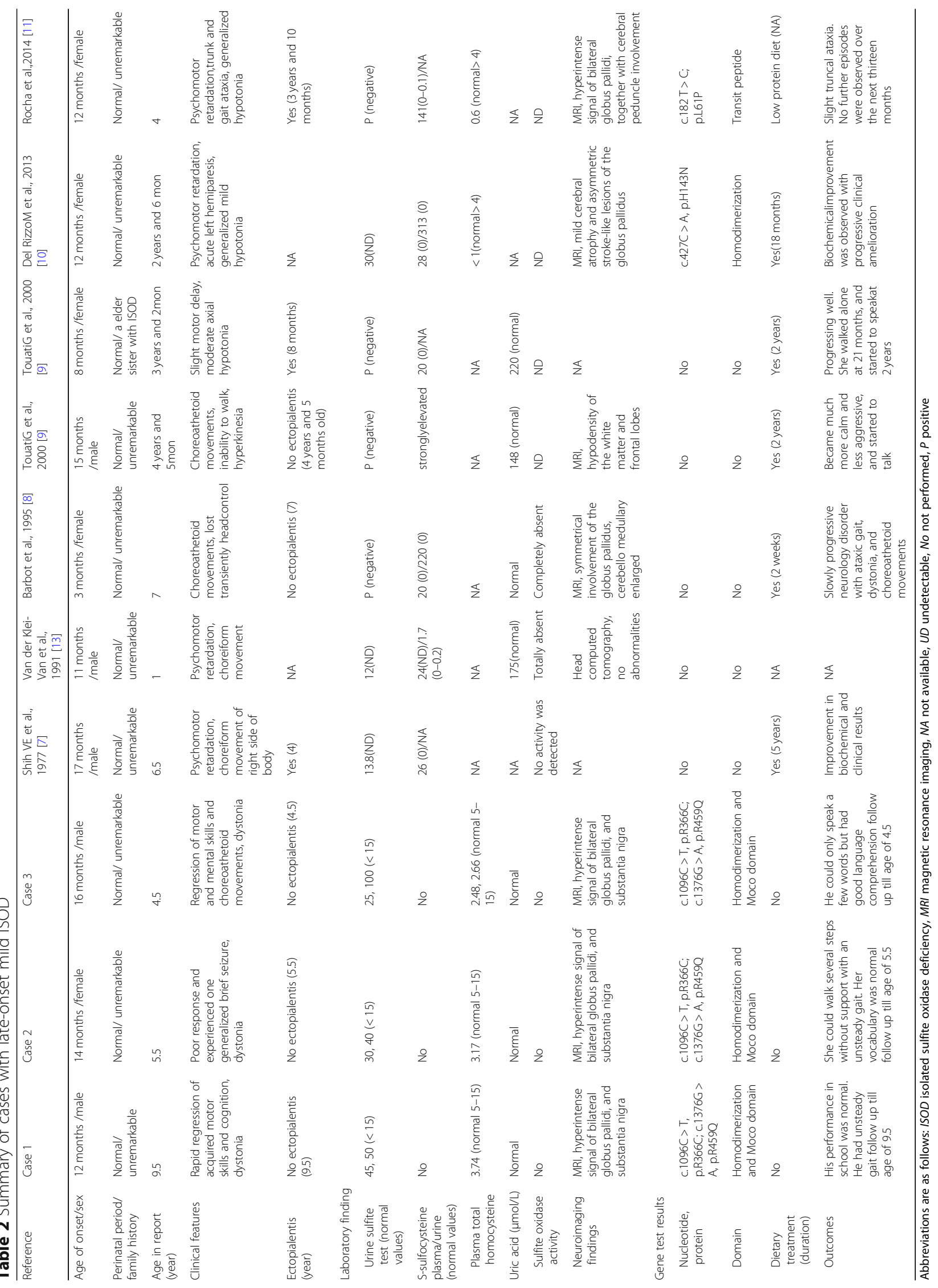


been reported in the literature [7-11, 13]. The clinical features of these patients, including our cases, are listed in Table 2. We have summarized several characteristics of late-onset mild ISOD: older age of onset (mean age, 12 months); seizures (1/10) and ectopia lentis (3/10) are rarely observed; normal development before disease onset (10/10); monophasic clinical course (9/10); and patients are more prone to bilateral globus pallidus and/or substantia nigra involvement (6/10).

Mechanisms for milder presentations of ISOD have not been fully elucidated. It has been reported that patients with missense mutations in the SUOX gene have a milder clinical presentation than those with null mutations [2], since missense mutations of the SUOX gene only lead to decreased biosynthesis of SUOX, whereas null mutations of the SUOX gene lead to complete abolishment of SUOX biosynthesis [11]. The genetic data from the case series presented here and from 2 cases of mild ISOD reported by others indicated the presence of missense mutations in the SUOX gene, further supporting the hypothesis that missense mutations of the SUOX gene contribute to a milder presentation.

Our case series and 2 cases of late-onset mild ISOD reported by others show decreased homocysteine levels. Sulfite reacts readily with free thiol groups to form sulfocysteine. It can also conjugate with homocysteine to form sulfocysteine, thus depleting plasma homocysteine [2]. Therefore, decreased homocysteine levels may facilitate a diagnosis of ISOD [11]. The slightly decreased levels of homocysteine were ignored by our clinicians before the results from exome sequencing for this family were made available. Therefore, we suggest that physicians need to be aware that decreased levels of homocysteine are observed in late-onset mild ISOD.

More than $90 \%$ of patients with classic early-onset ISOD show severe cerebral and cerebellar atrophy and/ or cystic white matter changes [2, 14, 15]. However, brain MRI scans of mild cases, including the three cases reported here, showed lesions mainly in the bilateral globus pallidus and substantia nigra at the acute stage, and later, the lesions improved and stabilized, suggesting that this pattern of bilateral globus pallidus and/or substantia nigra damage might be specific to mild ISOD. This imaging pattern may explain why patients in this cohort mainly present with motor regression and movement disorders rather than seizures and developmental delays. Neuro-imaging patterns of the symmetric basal ganglia and the brain stem have etiological diversity in children. Similar neuro-imaging findings are observed both in inherited metabolic diseases and acquired disorders, including Leigh syndrome, maple syrup urine disease, Wilson disease, organic acidurias, succinic semialdehyde dehydrogenase deficiency, kernicterus, and carbon monoxide poisoning. Most of these inherited metabolic diseases are progressively aggravated over the clinic course, and regular metabolic screening may provide diagnostic indicators. Furthermore, identification of acquired etiologies would be realized in cases of kernicterus and carbon monoxide poisoning. Symmetric basal ganglia involvement with low plasma homocysteine with or without dislocated lens would be important clues for the diagnosis of ISOD, especially the late-onset mild form.

Restricting dietary intake of methionine, cysteine, and taurine has been reported to be effective in patients with mild ISOD [7-11]. Touati et al. reported that when two children in a family were affected, the prognosis of the child who used dietary therapy was better than that of the child who did not [9]. However, clinical and neuroimaging improvements were observed in our patients even though dietary therapy was not used, suggesting that late-onset mild ISOD might spontaneously recover in some circumstances. Future studies to elucidate the mechanistic link between the genotype and phenotype of the disease and outcomes of patients with ISOD are required to gain better insight.

In conclusion, to our knowledge, this is the first literature review to summarize the characteristics of lateonset mild ISOD and the first report of late-onset mild ISOD cases with a stable clinical course and spontaneous recovery without dietary therapy. We propose that when patients present with late-onset symptoms, a monophasic clinical course, neuroimaging indicating a bilateral globus pallidus lesion, and decreased homocysteine levels, ISOD should be considered. Furthermore, we can reasonably predict a good prognosis for children with late-onset mild ISOD based on their monophasic clinical course and reversible neuroimaging features.

\section{Supplementary information}

Supplementary information accompanies this paper at https://doi.org/10. 1186/s12887-019-1889-5.

Additional file 1: Video S1. Video for case 1 at the age of 9.5 years showing the movement ability. The boy had unsteady gait, no choreiform movements were observed.

Additional file 2: Video S2. Video for case 2 at the age of 5.5 years showing the movement ability The girl could walk several steps without support, with an unsteady gait and choreiform movements.

Additional file 3: Video S3. Video for case 2 at the age of 5.5 years showing the language ability. Her vocabulary was normal, she could read smoothly.

Additional file 4: Video S4. Video for case 3 at the age of 4.5 years showing the movement ability. He could not sit until age 4.5 years because of generalized hypertonia. He had some mild choreiform movements.

\section{Abbreviations}

CSF: Cerebrospinal fluid; FLAIR: Fluid-attenuated inversion recovery; ISOD: Isolated sulfite oxidase deficiency; MRI: Magnetic resonance imaging; SUOX: Sulfite oxidase gene; SUOX: Sulfite oxidase protein; T2WI: T2-weighted 


\section{Acknowledgements}

We would like to thank all the patients and parents for participating in this study. We would like to thank Xiaomei Shu MD form the Department of Pediatrics, Affiliated Hospital of Zunyi Medical University, China, for helping in establishing the diagnosis

\section{Author contributions}

MT: main author and initiator of the study. SL, LH and XS: literature search and data analysis. FZ and JY: biochemical detection. DM: substantial financial support provided. YQ: conceived and designed the study. All authors read and approved the final manuscript

\section{Funding}

The research of this study is funded by the National Science Foundation of China $(81630038,81771634,81842011,81971433,81971428)$ and the National Key Project of Neonatal Children (1311200003303). The funders had no role in study design, data collection and analysis, and preparation of the manuscript.

\section{Availability of data and materials}

All data generated or analyzed during this study are included in this article.

\section{Ethics approval and consent to participate}

This study was approved by the Ethics committee of the Affiliated Hospital of Zunyi Medical University, China. Written informed consent for genetic testing and participation in this study were obtained from the parents of the patients.

\section{Consent for publication}

Written informed consent for publication of their clinical details and clinical images (videos) were obtained from the parents of the patients. A copy of the consent form is available for review by the editor of this journal.

\section{Competing interests}

The authors declare that they have no competing interests.

\section{Author details}

'Department of Pediatrics, West China Second University Hospital, Sichuan University, Chengdu 610041, China. ${ }^{2}$ Key Laboratory of Birth Defects and Related Diseases of Women and Children, (Sichuan University), Ministry of Education, Chengdu 610041, China. ${ }^{3}$ Department of Pediatrics, Affiliated Hospital of Zunyi Medical University, Zunyi 563003, China. ${ }^{4}$ West China College of Stomatology, Sichuan University, Chengdu 610041, China.

Received: 3 March 2019 Accepted: 15 December 2019

Published online: 23 December 2019

\section{References}

1. Irreverre F, Mudd SH, Heizer WD, Laster L. Sulfite oxidase deficiency: studies of a patient with mental retardation, dislocated ocular lenses, and abnormal urinary excretion of S-sulfo-l-cysteine, sulfite, and thiosulfate. Biochem Med. 1967;1:187-217.

2. Claerhout $H$, Witters $P$, Regal $L$, Jansen $K$, Van Hoestenberghe MR, Breckpot J. Vermeersch, P. isolated sulfite oxidase deficiency. J Inherit Metab Dis. 2018;41(1):101-8. https://doi.org/10.1007/s10545-017-0089-4.

3. Kisker C, Schindelin H, Pacheco A, Wehbi WA, Garrett RM, Rajagopalan KV, et al. Molecular basis of sulfite oxidase deficiency from the structure of sulfite oxidase. Cell. 1997:91:973-83.

4. Rupar CA, Gillett J, Gordon BA, Ramsay DA, Johnson JL, Garrett RM, et al. Isolated sulfite oxidase deficiency. Neuropediatrics. 1996;27:299-304. https:// doi.org/10.1055/s-2007-973798.

5. Garrett RM, Johnson JL, Graf TN, Feigenbaum A, Rajagopalan KV. Human sulfite oxidase R160Q: identification of the mutation in a sulfite oxidasedeficient patient and expression and characterization of the mutant enzyme. Proc Natl Acad Sci U S A. 1998;95(11):6394-8.

6. Tan WH, Eichler FS, Hoda S, Lee MS, Baris H, Hanley CA, et al. Isolated sulfite oxidase deficiency: a case report with a novel mutation and review of the literature. Pediatrics. 2005;116:757-66. https://doi.org/10.1542/peds.2004-1897.

7. Shih VE, Abroms IF, Johnson JL, Carney M, Mandell R, Robb RM, et al. Sulfite oxidase deficiency. Biochemical and clinical investigations of a hereditary metabolic disorder in sulfur metabolism. N Engl J Med. 1977;297(19):1022-8. https://doi.org/10.1056/NEJM197711102971902.

8. Barbot C, Martins E, Vilarinho L, Dorche C, Cardoso ML. A mild form of infantile isolated sulphite oxidase deficiency. Neuropediatrics. 1995;26(6): 322-4. https://doi.org/10.1055/s-2007-979783.

9. Touati G, Rusthoven E, Depondt E, Dorche C, Duran M, Heron B, et al. Dietary therapy in two patients with a mild form of sulphite oxidase deficiency. Evidence for clinical and biological improvement. J Inherit Metab Dis. 2000;23:45-53.

10. Del RM, Burlina AP, Sass JO, Beermann F, Zanco C, Cazzorla C, et al. Metabolic stroke in a late-onset form of isolated sulfite oxidase deficiciency. Mol Genet Metab. 2013;108:263-6. https://doi.org/10.1016/j.ymgme.2013.01.011.

11. Rocha S, Ferreira AC, Dias Al, Vieira JP, Sequeira S. Sulfite oxidase deficiencyan unusual late and mild presentation. Brain and Development. 2014;36: 176-9. https://doi.org/10.1016/j.braindev. 2013.01.013.

12. Richards S, Aziz N, Bale S, Bick D, Das S, Gastier-Foster J, et al. Standards and guidelines for the interpretation of sequence variants: a joint consensus recommendation of the american college of medical genetics and genomics and the association for molecular pathology. Genet Med. 2015; 17(5):405-24. https://doi.org/10.1038/gim.2015.30.

13. van der Klei-van MJM, Smit LM, Brockstedt M, Jakobs C, Dorche C, et al. Infantile isolated sulphite oxidase deficiency: report of a case with negative sulphite test and normal sulphate excretion. Eur J Pediatr. 1991;150(3):196-7.

14. Hoffmann C, Ben-Zeev B, Anikster Y, Nissenkorn A, Brand N, Kuint J, et al. Magnetic resonance imaging and magnetic resonance spectroscopy in isolated sulfite oxidase deficiency. J Child Neurol. 2007;22:1214-21. https:// doi.org/10.1177/0883073807306260.

15. Bosley TM, Alorainy IA, Oystreck DT, Hellani AM, Seidahmed MZ, Osman Mel $F$, et al. Neurologic injury in isolated sulfite oxidase deficiency. Can J Neurol Sci. 2014;41(1):42-8

\section{Publisher's Note}

Springer Nature remains neutral with regard to jurisdictional claims in published maps and institutional affiliations.
Ready to submit your research? Choose BMC and benefit from:

- fast, convenient online submission

- thorough peer review by experienced researchers in your field

- rapid publication on acceptance

- support for research data, including large and complex data types

- gold Open Access which fosters wider collaboration and increased citations

- maximum visibility for your research: over $100 \mathrm{M}$ website views per year

At BMC, research is always in progress.

Learn more biomedcentral.com/submission 